\title{
Sistema reproductivo de Trichopetalum plumosum (Ruiz \& Pav.) J.F. Macbr. (Asparagaceae), geófita endémica de Chile
}

\section{Breeding system of Trichopetalum plumosum (Ruiz \& Pav.) J.F. Macbr. (Asparagaceae), geophyte endemic to Chile}

\author{
Marcela Cuartas-Domínguez ${ }^{1,2,3^{*}}$, Alejandra Rojas-Céspedes ${ }^{1,2}$, Paola Jara-Arancio $^{1,4}$ \& Mary T. \\ K. ARROYO ${ }^{1,2}$ \\ ${ }^{1}$ Instituto de Ecología y Biodiversidad (IEB), Facultad de Ciencias, Universidad de Chile, Santiago, Chile. \\ ${ }^{2}$ Departamento de Ciencias Ecológicas, Universidad de Chile, Ñuñoa, Santiago, Chile. \\ ${ }^{3}$ Grupo de Investigación en Orquídeas, Ecología y Sistemática Vegetal. Universidad Nacional de Colombia, Sede Palmira, \\ Colombia. \\ ${ }^{4}$ Departamento de Ciencias Biológicas y Departamento de Ecología y Biodiversidad, Universidad Andrés Bello, Santiago, \\ Chile. \\ *mcuartasd@ug.uchile.cl, marcelcd28@yahoo.es
}

\begin{abstract}
RESUMEN
En este estudio se evaluó el sistema reproductivo de Trichopetalum plumosum (Ruiz \& Pav.) J.F. Macbr. (Asparagaceae), una geófita endémica de Chile. Se realizaron cinco tratamientos de polinización controlada (agamospermia, autogamia autónoma, autopolinización, polinización cruzada y natural / control). Ninguna de las flores emasculadas y sin polinizar produjo frutos, lo que indica que esta especie no es apomíctica y, por lo tanto, necesita polen para la producción de semillas. La fructificación en el tratamiento de polinización cruzada $(70,0 \%)$ fue alta y similar al porcentaje alcanzado a través de la polinización natural (90,9\%). A pesar del porcentaje menor en la prueba de autopolinización (43,3\%), la autogamia autónoma $(90,0 \%)$ se acercó a los altos valores alcanzados en los dos primeros tratamientos mencionados. Para el promedio de semillas por flor tratada, los resultados concuerdan con los de fructificación. Sin embargo, al evaluar el número promedio de semillas por fruto formado, los tratamientos no mostraron diferencias significativas. Los resultados indican que la población de $T$. plumosum es autocompatible con una alta capacidad para la autogamia autónoma. Se sugiere que esta última se produce por el movimiento de los estambres hacia el estigma al final del día a medida que las flores se marchitan y cierran, correspondiendo a un modo de autofecundación tardía.
\end{abstract}

Palabras clave: Autocompatibilidad, autogamia autónoma, autofecundación tardía, geófita, subfamilia Lomandroideae.

\begin{abstract}
The breeding system of the geophyte endemic to Chile Trichopetalum plumosum (Ruiz \& Pav.) J.F. Macbr. (Asparagaceae) was evaluated with five controlled pollination treatments (agamospermy, autonomous autogamy, self-pollination, crosspollination and natural / control). Emasculated and unpollinated flowers did not produce fruits, therefore, this is a nonapomictic species and needs pollen for seed production. Fruit production via cross and natural pollination treatments were high with 70.0 and $90.9 \%$, respectively. Despite the low production via self-pollination (43.3\%), autonomous autogamy $(90.0 \%)$ achieved high values as cross and natural pollination treatments. The results for the mean number of seeds per flower were similar to fruiting. However, analysis of the mean number of seeds per fruit did not show significant differences. These results indicate that the population of T. plumosum is self-compatible with a high capacity for autonomous autogamy. It could be possible that autonomous autogamy of this species occurs when the stamens of the flowers move toward the stigma while they close and wither at the end of the day, corresponding to a delayed selfing mode.
\end{abstract}

KEYWORDS: Self-compatibility, autonomous autogamy, delayed selfing, geophyte, subfamily Lomandroideae. 


\section{INTRODUCCIÓN}

El sistema reproductivo de las plantas depende de factores intrínsecos (p.ej., inercia filogenética, rasgos de historia de vida) y extrínsecos (p.ej., factores abióticos e interacciones bióticas) que en conjunto modulan su éxito reproductivo (Barrett \& Harder 1996). Los sistemas de reproducción varían desde la autopolinización no asistida (espontánea) o autogamia autónoma, hasta la polinización cruzada o xenogamia (Barrett \& Harder 1996). La autogamia (autónoma o facilitada) se manifiesta en plantas genéticamente autocompatibles, en las que la disposición de las anteras y del estigma permite el depósito de polen espontáneo o mediado por un vector. Por su parte, la xenogamia requiere de un vector de polinización para el intercambio de polen entre distintos individuos. Siendo la autofertilización versus la fertilización cruzada uno de los temas principales de investigación dentro del estudio sobre la evolución de las estrategias reproductivas en plantas (Barrett 1998, Holsinger 2000, Goodwillie et al. 2005, Johnston et al. 2009, Igic \& Busch 2013). Estas dos estrategias presentan ventajas y desafíos; por un lado, la autofertilización puede promover el aseguramiento reproductivo en ambientes donde los vectores de polen o individuos conespecíficos sean escasos, pero puede conllevar depresión por endogamia y disminución de la variabilidad genética en las distintas poblaciones; mientras que la polinización cruzada aumenta la diversidad genética, pero siempre es dependiente de un vector biótico o abiótico y de individuos conespecíficos (Barrett 1998, Holsinger 2000, Barrett 2003).

La mayoría de las angiospermas son hermafroditas y tienen diversas adaptaciones florales que potencian la fecundación cruzada y limitan los efectos nocivos de la endogamia (Barrett \& Harder 1996, Holsinger 2000). Sin embargo, a pesar de las ventajas de la xenogamia, el cambio a la autofecundación es una transición evolutiva frecuente entre las estrategias de apareamiento de las plantas (Holsinger 2000, Igic et al. 2008). Determinar las fuerzas selectivas responsables para la evolución de una estrategia sobre la otra, o el mantenimiento de estrategias mezcladas estables, es uno de los objetivos principales dentro de la teoría evolutiva de las plantas con flores (Barrett 1998, Vogler \& Kalisz 2001, Goodwillie et al. 2005, Eckert et al. 2006, Johnston et al. 2009, Busch \& Delph 2012).

La autofertilización se da en especies autocompatibles a través de la autogamia ya sea facilitada o autónoma, y potencialmente asegura la reproducción (Goodwillie et al. 2005, Busch \& Delph 2012). Por otro lado, en especies autoincompatibles, la reproducción se lleva a cabo principalmente a través de fertilización cruzada, aumentando la dependencia de vectores para la polinización (Barrett 1998, Goodwillie et al. 2005). Esto último puede tener consecuencias negativas, especialmente para especies polinizadas por animales y marcadamente autoincompatibles, tanto en ambientes estresantes como en hábitats perturbados por la acción antrópica (González \& Pérez 2010, Eckert et al. 2010). Eckert et al. (2010) compararon los sistemas reproductivos de plantas provenientes de hábitats perturbados y levemente perturbados, donde observaron que la tasa de cruzamiento es significativamente más baja en hábitats perturbados. La reducción del polen cruzado causada por perturbación humana reduce la producción de semillas en especies estrictamente autoincompatibles, mientras que en especies autocompatibles con capacidad para autogamia autónoma, podría producirse un complemento total de semillas en su mayoría autofertilizadas (Larson \& Barrett 2000, Eckert et al. 2010). En cuanto a hábitats estresantes, como las zonas muy secas o desérticas, se ha detectado que las especies sometidas a dichos hábitats pueden tener altas tasas de autofertilización, adquiridas a lo largo de su historia evolutiva debido a las presiones selectivas ambientales (Pérez et al. 2009, González \& Pérez 2010). La autofertilización, ya sea en ambientes estresantes o perturbados por la acción antrópica, potencialmente proporcionaría aseguramiento reproductivo mediante la compensación por escasez de polen cruzado (Goodwillie et al. 2005, González \& Pérez 2010, Eckert et al. 2010).

Existe escasa información sobre los sistemas de reproducción en muchas angiospermas (Ferrer \& Good 2012). De un total aproximado de 268.500 especies, sólo se ha caracterizado el sistema reproductivo en 5.609 de éstas (Ferrer \& Good 2012). También hay un gran desconocimiento de los sistemas reproductivos en algunas formas de vida, como es el caso de las geófitas, sobre las cuales se han realizado muy pocos estudios a nivel mundial (Saavedra et al. 1996, Cavieres \& Arroyo 1999, Jesson \& Barrett 2002, Arroyo et al. 2002, Kleizen et al. 2008, Geerts \& Pauw 2012).

El género sudamericano Trichopetalum, con dos especies: T. plumosum (Ruiz \& Pav.) J.F. Macbr. y T. chosmalensis Guagl. \& Belgrano (Marais \& Reilly 1978, Guaglianone \& Belgrano 2003), pertenece a la familia Asparagaceae, subfamilia Lomandroideae; esta última agrupa 14 géneros y 178 especies (Stevens 2001, APG III 2009, Chase et al. 2009). En esta subfamilia se ha descrito autoincompatibilidad genética para algunas especies del género Cordyline (Beever 1981, Beever 1983, Beever \& Parkes 1996, Hinkle 2007) y autocompatibilidad en dos especies del género Laxmannia (James et al. 1999). Dado que $T$. plumosum es una hierba perenne, se espera que sea mayormente autocompatible, pues existe una tendencia significativa a nivel mundial, que indica que las especies herbáceas son principalmente autocompatibles (Raduski et al. 2012). Para evaluar esta hipótesis se planteó como objetivo determinar el sistema reproductivo de T. plumosum, mediante pruebas de polinización controladas. 


\section{MATERIALES Y MÉTODOS}

SiTIO Y ESPECIE EN ESTUDIO

El sitio de estudio se encuentra en la vertiente norte del Cerro Calán (3323'41'S, 70³2’04'O), Santiago, Región Metropolitana, Chile, a 820 m s.n.m. El clima del área de estudio es de tipo mediterráneo (Di Castri \& Hajek 1976) con precipitaciones de invierno. En un año normal la cantidad total de precipitaciones en el valle de Santiago es de alrededor de $350 \mathrm{~mm}$, aunque existe una alta variabilidad interanual (Aceituno 1990). La temperatura media anual es de $14{ }^{\circ} \mathrm{C}$, con una moderada oscilación térmica entre el período invernal y la época estival (Di Castri \& Hajek 1976). El estudio fue llevado a cabo en la primavera del año 2014, abarcando los meses de septiembre a noviembre, con temperaturas extremas máximas de $32{ }^{\circ} \mathrm{C}$ (Dirección Meteorológica de Chile-sitio web).

Trichopetalum plumosum es una especie geófita, endémica de Chile, de matorral y bosque esclerófilo (Marais \& Reilly 1978, Teillier et al. 2005), que se distribuye desde la región de Coquimbo hasta la Araucanía, incluyendo la Metropolitana $\left(29^{\circ}-38^{\circ} \mathrm{S}\right)$ (Fig. 1a), desde el nivel del mar hasta alrededor de $1.500 \mathrm{~m}$ s.n.m. en la vertiente occidental de los Andes (Zuloaga et al. 2008). Su distribución abarca la región de Chile central, donde se encuentra una rica y diversa flora nativa, que crece en un gradiente de aridez severo (Arroyo et al. 1995). Esta especie habitualmente habita suelos arenosos, muy secos, de alta evaporación, donde el agua escurre rápidamente. Es por esta razón que los umbrales de hidratación que requiere para germinar son altos y se asocian a una sumatoria de pulsos variables de precipitación que aseguran el completo desarrollo de su ciclo biológico (Jara-Arancio et al. 2006).

Esta geófita tiene tubérculos alargados, con tallos sencillos de 10 a $20 \mathrm{~cm}$ de alto; hojas lineares, puntiagudas, de 10 a $25 \mathrm{~cm}$ de largo y 1 a $5 \mathrm{~mm}$ de ancho, glaucas. Las flores se agrupan en racimos laxos; miden de 10 a $15 \mathrm{~mm}$ de diámetro, son blanco-verdosas y están formadas por seis tépalos dispuestos en dos series, con tres exteriores estriados y los interiores tapizados con pelos blancos (Fig. 1b). Seis estambres que nacen del extremo inferior de los tépalos; filamentos glabros; anteras cuya dehiscencia se abre hacia el eje de la flor. Ovario trilocular; estilo recto; estigma trilobulado. Cápsula cartilaginosa; ápice mucronado que representa al estilo persistente. Semillas subreniformes, negras, brillantes. Florece desde septiembre hasta noviembre (Navas 1973, Hoffmann 1989, Muñoz \& Moreira 2000, Riedemann \& Aldunate 2001). Las flores individuales abren en las primeras horas de la mañana (07:00 - 09:00 h), y se marchitan y cierran en horas de la tarde (17:00 19:00 h) (Fig. 1c); por lo que la duración de cada flor es de aproximadamente $12 \mathrm{~h}$ (observaciones personales).

En la población de estudio se observaron diferentes insectos visitando y tocando estructuras reproductivas, dentro de los cuales se lograron identificar: escarabajos (Lichnia limbata Erichson (Scarabaeidae) y Epiclines gayi Chevrolat (Cleridae)); algunos sírfidos como Allograpta sp. (Syrphidae); una especie de mosquito no determinada (Culicidae); y al menos dos especies de abejas de la familia Halictidae.

\section{SisTEMA REPRODUCTIVO}

Para determinar el sistema reproductivo de T. plumosum, las inflorescencias de 115 plantas fueron cubiertas con bolsas de tul (Figs. 1d, 1e), antes de la antesis de sus flores, para evitar la llegada de polinizadores. Posteriormente, se llevaron a cabo cuatro tratamientos de polinización más uno de polinización natural (tratamiento control) (Kearns \& Inouye 1993, Eckert et al. 2010). Los tratamientos fueron los siguientes: (a) Agamospermia: para evaluar si esta especie puede producir semillas sin la intervención de polen (apomixis), se emasculó una flor recién abierta por planta $(n=25)$, esto se realizó antes de la dehiscencia de las anteras, y luego se volvió a cubrir la inflorescencia; (b) Autogamia autónoma: para evaluar si esta planta es capaz de producir semillas sin la intervención de polinizadores (autofertilización autónoma), se dejaron yemas florales sin emascular y cubiertas con bolsas de tul hasta la cosecha de semillas (una yema por planta, $n=30$ ); (c) Autopolinización: para probar si la planta es capaz de producir semillas con polen del mismo individuo transportado por polinizadores (autogamia facilitada: polen de la misma flor, o geitonogamia: polen desde otra flor), se realizó un tratamiento de autopolinización manual $(n=$ 30); es decir, una vez abierta la flor y cuando el estigma estuvo receptivo (brillante y viscoso), se quitó la bolsa y se polinizó con el polen de la misma planta, después se volvió a cubrir la inflorescencia hasta la recolección de los frutos; (d) Polinización cruzada: para probar si la planta es capaz de producir semillas con polen de otras plantas (xenogamia), se realizó un tratamiento de polinización cruzada manual, mediante el cual se emascularon 30 flores que, en el momento en que el estigma estuvo receptivo, fueron polinizadas con polen proveniente de diferentes individuos de la misma especie que se encontraran a una distancia $\geq$ a 10 $\mathrm{m}$, para evitar posible consanguinidad entre los individuos cercanos y la planta experimental, luego se volvió a cubrir la inflorescencia hasta la recolección de los frutos; (e) Polinización natural (control): para evaluar la polinización en condiciones naturales se marcaron de manera discreta 22 yemas florales, una por planta, se dejaron sin cubrir y fueron recolectadas en el estado de fruto (Tabla 1).

Durante cinco días consecutivos se estableció todo el montaje experimental; cada clase de tratamiento se realizó el mismo día. Se llevó a cabo un solo tratamiento por planta ( $n$ $\approx 30$ ), considerando una flor. Después de cuatro semanas se procedió a la recolección de las flores tratadas, ya fuera que estuvieran en estado de fruto o como flores secas, y fueron 
llevadas al laboratorio para contabilizar la producción de semillas por fruto formado.

\section{ANÁLISIS DE DATOS}

La producción de frutos (fruto versus no-fruto) para todos los tratamientos fue comparada estadísticamente utilizando una prueba Chi-cuadrado de independencia (Zar 2010). Dado que los datos para la producción de semillas por fruto formado presentaron una distribución normal (prueba de Kolmorogov-Smirnov, $d=0,10 ; P>0,20$ ) y homocedasticidad de varianzas, se comparó la producción promedio de semillas por fruto para los tratamientos (excluyendo agamospermia, pues no se produjeron frutos ni semillas en las flores emasculadas y embolsadas) con una ANOVA de una vía de efectos fijos (Zar 2010). La producción de semillas por flor tratada experimentalmente no presentó una distribución normal (prueba de KolmorogovSmirnov, $d=0,27 ; P<0,01)$. Por lo tanto, los datos para los tratamientos (excluyendo agamospermia) fueron analizados con una prueba de Kruskal-Wallis, realizando comparaciones múltiples a posteriori de los rangos promedio para todos los grupos (Siegel \& Castellan 1988, StatSoft 2004, Zar 2010). Todos los análisis estadísticos fueron llevados a cabo en el programa STATISTICA 7.0 para Windows (StatSoft 2004).

Adicionalmente, se calculó el Índice de AutoFertilidad (IAF $=$ producción de semillas (o frutos) de plantas aisladas / producción de semillas (o frutos) de polinización cruzada artificial), este índice varía continuamente desde cero (i.e., no hay autofertilidad) a valores por encima de uno (altos niveles de autofertilidad) (Lloyd \& Schoen 1992). Para los datos obtenidos, este índice se calculó dividiendo el número promedio de semillas producidas por flor aislada (resultado del tratamiento de autogamia autónoma) por el número promedio de semillas producidas por flor tratada después de polinización cruzada manual.
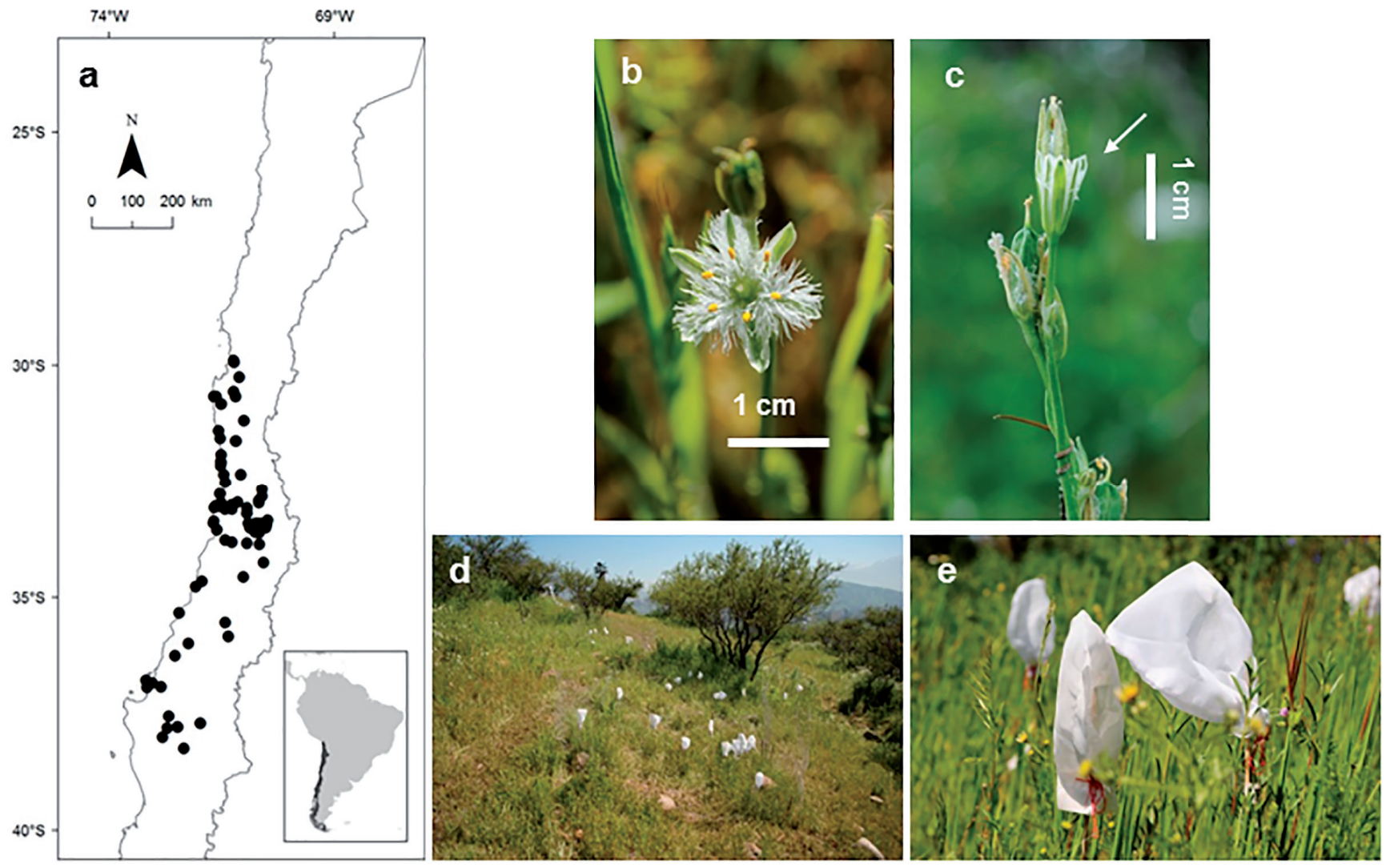

Figura 1. (a) Distribución de Trichopetalum plumosum en Chile, donde los círculos negros son ocurrencias georreferenciadas. (b) Detalle de una flor completamente abierta en las primeras horas del día (07:00 - 09:00 h). (c) Flor senescente (17:00 - 19:00 h). (d) Hábitat y montaje experimental. (e) Inflorescencias cubiertas con bolsas de tul. Fotografías por Karina Bacelli. / (a) Trichopetalum plumosum distribution in Chile, black circles are georeferenced occurrences. (b) Flower completely open in the early hours of the day (07:00 - 09:00 h). (c) Senescence of flower (17:00 - 19:00 h). (d) Habitat and experimental setup. (e) Bagged inflorescences with tulle. Photos by Karina Bacelli. 
TABla 1. Tratamientos de polinización controlada aplicados a 137 flores de T. plumosum, una flor por planta. EE = Error estándar. / Controlled pollination treatments to 137 flowers of T. plumosum, a flower per plant. $S E=$ Standard error.

\begin{tabular}{|c|c|c|c|c|}
\hline Tratamiento & $\begin{array}{c}\mathrm{N}^{\mathrm{o}} \text { FLORES POR } \\
\text { TRATAMIENTO }\end{array}$ & $\begin{array}{c}\% \text { FRUTOS POR } \\
\text { TRATAMIENTO } \\
\left(\mathrm{N}^{\mathrm{o}} \text { FRUTOS TOTALES }\right)^{\dagger}\end{array}$ & $\begin{array}{c}\mathrm{N}^{\mathrm{o}} \text { SEMILLAS POR } \\
\text { FRUTO FORMADO }^{\dagger \dagger} \\
\overline{\mathrm{X}} \pm E E\end{array}$ & $\begin{array}{c}\mathrm{N}^{\circ} \text { SEMILLAS POR } \\
\text { FLOR TRATADA } \\
\overline{\mathrm{X}}_{ \pm E E}\end{array}$ \\
\hline Agamospermia & 25 & $0,0(0)$ & $0,0 \pm 0,0$ & $0,0 \pm 0,0$ \\
\hline Autogamia autónoma & 30 & $90,0(27)$ & $29,9 \pm 2,2$ & $26,9 \pm 2,6^{\mathrm{a}}$ \\
\hline Autopolinización & 30 & $43,3(13)$ & $27,3 \pm 2,8$ & $11,8 \pm 2,8^{b}$ \\
\hline Polinización cruzada & 30 & $70,0(21)$ & $34,8 \pm 1,5$ & $24,4 \pm 3,2^{\mathrm{a}}$ \\
\hline $\begin{array}{l}\text { Polinización natural } \\
\text { (control) }\end{array}$ & 22 & $90,9(20)$ & $31,4 \pm 2,2$ & $28,5 \pm 2,8^{\mathrm{a}}$ \\
\hline
\end{tabular}

${ }^{\dagger}$ La fructificación de las flores tratadas no es independiente del tratamiento aplicado (Chi-cuadrado $=61,76 ;$ g.l. $=4 ; P=0,00$ ). $/$ Fruiting of treated flowers is not independent of the treatment applied (Chi-square test $=61.76$; d.f. $=4 ; P=0.00$ ).

${ }^{\dagger}$ No hay diferencias estadísticamente significativas entre tratamientos, según lo determinado por la prueba de ANOVA de una vía de efectos fijos $\left(F_{3,77}=1,80 ; P=0,15\right)$. / There is no significant statistical differences between treatments, according to ANOVA one-way fixed effects $\left(F_{3,77}=1.80 ; P=0.15\right)$.

+Letras distintas indican diferencias estadísticamente significativas según la prueba de Kruskal-Wallis con comparaciones múltiples $a$ posteriori de los rangos promedio para todos los tratamientos $(P<0,05)$. / Different letters indicate statistically significant difference, according to Kruskal-Wallis test with post hoc multiple comparisons of average ranks for all treatments $(P<0.05)$.

\section{RESULTADOS}

Ninguna de las flores emasculadas y sin polinizar $(n=25$, una flor por planta) produjo frutos (Tabla 1, Fig. 2). A partir del análisis de la tabla de contingencia 2 x 5 , se concluyó que se debe rechazar la hipótesis de independencia y por lo tanto, asumir que existe relación entre la fructificación y el tratamiento aplicado (Chi-cuadrado $=61,76$; g.l. $=4$; $P=0,00)$. Al realizar un análisis exploratorio adicional, subdividiendo la tabla de contingencia, para indagar sobre qué tratamientos estarían causando la significancia de la Chi-cuadrado calculada (Zar 2010) se encontró evidencia que sugiere que la producción de frutos en los tratamientos de agamospermia y autopolinización difieren de los otros tres tratamientos (Tabla 1, Fig. 2).

El número de semillas por fruto formado no difirió significativamente entre los tratamientos $\left(F_{3,77}=1,80\right.$ $P=0,15$ ) (Tabla 1, Fig. 3). En cuanto a la producción de semillas por flor tratada, se encontró un efecto significativo del tratamiento de polinización sobre dicha producción (prueba de Kruskal-Wallis: $H=16,50$; g.l. $=3 ; P \leq 0,00$ ). El tratamiento con mayor producción de semillas por flor tratada fue el de polinización natural, seguido de autogamia autónoma y polinización cruzada, y finalmente el de autopolinización (Tabla 1). Las comparaciones múltiples a posteriori de los rangos promedio para los tratamientos indicaron que la producción de semillas en las flores autopolinizadas mostró diferencias estadísticamente significativas con respecto a las flores que: fueron cruzadas
$(P=0,02)$, se polinizaron de manera autógama $(P=$ $0,00)$ y de manera natural $(P=0,01)$. Los tratamientos de autogamia autónoma, polinización cruzada y natural no resultaron distintos entre sí (Tabla 1, Fig. 4). La proporción entre el número promedio de semillas producidas por flor aislada (tratamiento de autogamia autónoma) y el número promedio de semillas producidas por flor polinizada de manera cruzada (IAF) dio un valor de 1,10.

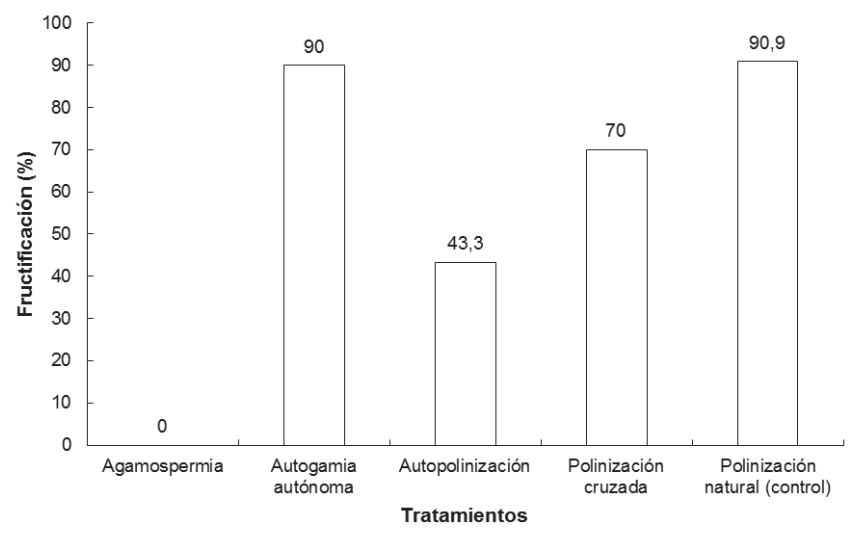

FiguRa 2. Porcentaje de fructificación de las flores tratadas, una flor por planta, bajo cada uno de los cinco tratamientos de polinización. Existe una relación entre la fructificación y el tratamiento aplicado (Prueba de Chi-cuadrado $=61,76 ;$ g.l. $=4 ; P=0,00$ ). $/$ Fruiting percentage of treated flowers, one flower per plant, under each pollination treatment. There is a relation between fruiting and treatments (Chi-square test $=61.76$; d.f. $=4 ; P=0.00$ ). 


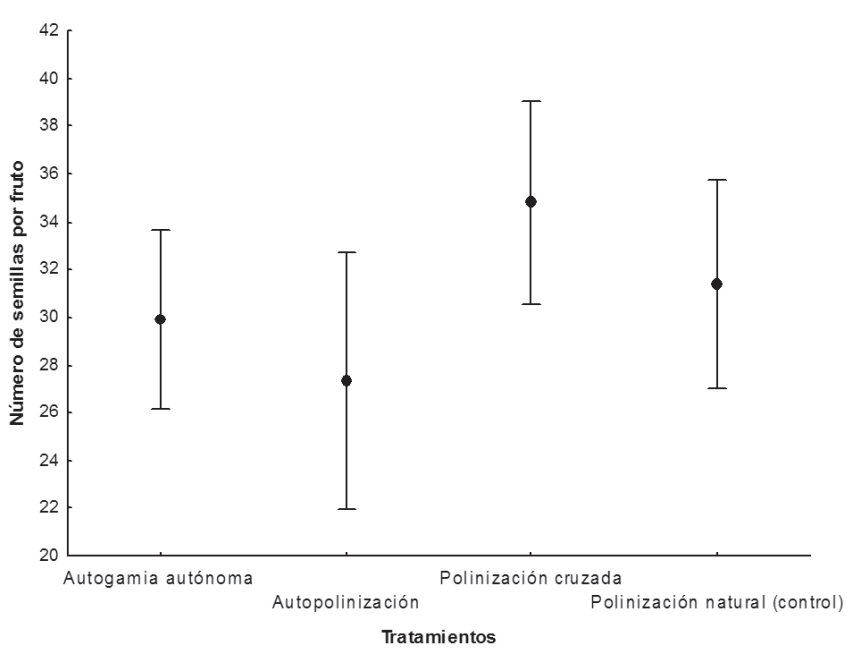

FIGURA 3. Número promedio de semillas por fruto formado, para cuatro tratamientos de polinización (se excluye agamospermia). No hay diferencias estadísticas significativas según la prueba de ANOVA de una vía de efectos fijos $\left(F_{3,77}=1,80 ; P=0,15\right)$. Las barras verticales indican los intervalos de confianza del $95 \%$. / Mean number of seeds per fruit formed, for four pollination treatments (agamospermy is excluded). There is no significant statistical differences by ANOVA one-way fixed effects $\left(F_{3,77}=\right.$ $1.80 ; P=0.15)$. Vertical bars denote $95 \%$ confidence intervals.

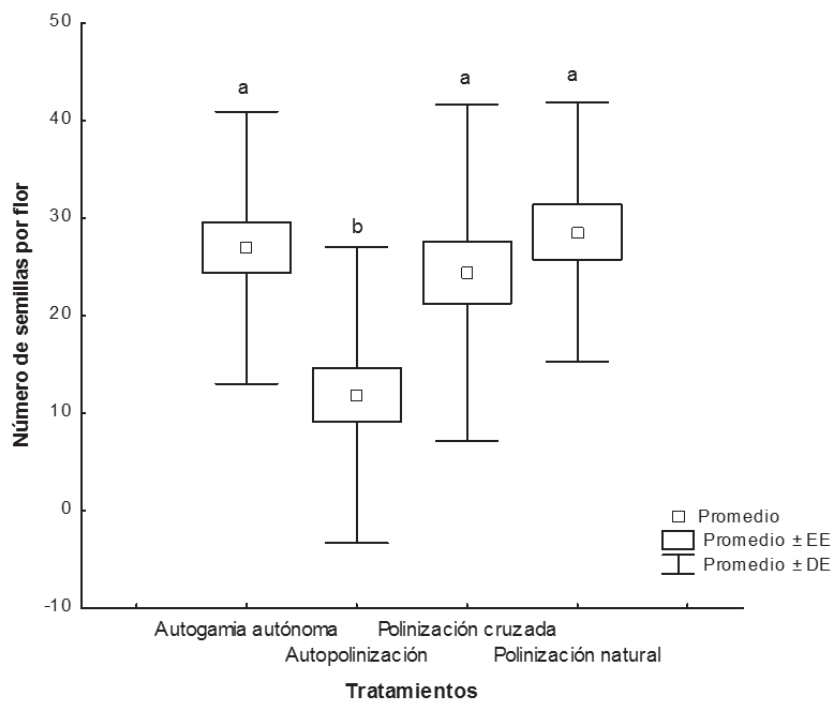

FIGURA 4. Número promedio de semillas por flor tratada, para cuatro tratamientos de polinización (se excluye agamospermia). Valores promedio con letras distintas indican diferencias estadísticamente significativas, según la prueba de Kruskal-Wallis (comparaciones múltiples a posteriori de los rangos promedio para todos los tratamientos, $P<0,05)$. / Mean number of seeds per treated flower, for four pollination treatments (agamospermy is excluded). Mean values with different letters indicate statistically significant difference, according to Kruskal-Wallis test (post hoc multiple comparisons of average ranks for all treatments, $P<0.05$ ).

\section{DISCUSIÓN}

La población estudiada de T. plumosum tiene una estrategia reproductiva mixta, donde la producción de semillas es el resultado tanto de polinización cruzada como de autofecundación (Goodwillie et al. 2005). Ninguna de las flores emasculadas y sin polinizar produjo frutos, indicando que $T$. plumosum necesita polen para producir semillas. Las diferencias encontradas entre el tratamiento de autopolinización con respecto a los otros tres tratamientos en cuanto a la fructificación y al número de semillas por flor tratada (Figs. 2 y 4), podrían deberse a la no correspondencia con los horarios de receptividad máxima del estigma al momento de autopolinizar las flores, más que a un efecto de autoincompatibilidad en T. plumosum. Esto último también se soporta por la falta de diferencias con respecto al número de semillas por fruto formado entre los cuatro tratamientos de polinización (Fig. 3).

La población de $T$. plumosum en Cerro Calán es autocompatible, pues tiene una alta capacidad para la autogamia autónoma y por consiguiente presenta altos niveles de autofertilidad. La autopolinización en plantas genéticamente autocompatibles podría ser mediada por polinizadores u ocurrir de manera espontánea (Lloyd \& Schoen 1992). En T. plumosum el estigma y las anteras se encuentran al mismo nivel en la flor. Al cerrar las flores en la tarde, las anteras toman contacto con el estigma, permitiendo así la autopolinización espontánea. De esta forma, la autogamia autónoma en T. plumosum corresponde a la llamada autogamia tardía (“delayed selfing"). Ésta ocurre cuando los movimientos de las partes florales al final de la antesis conducen a que haya contacto entre el polen y el estigma, provocando la fertilización de los óvulos que no han sido previamente fertilizados de manera cruzada (Lloyd \& Schoen 1992). La autogamia tardía es considerada un mecanismo de aseguramiento reproductivo en ambientes en donde las visitas de los polinizadores son altamente fluctuantes en el espacio y/o el tiempo o en donde las parejas potenciales son escasas (Lloyd \& Schoen 1992, Kalisz \& Vogler 2003, Pérez et al. 2009, González \& Pérez 2010). En el caso de T. plumosum, es probable que si las flores no reciben visitas de polinizadores durante el día, se asegura la producción de semillas a través de autogamia tardía en las últimas horas de vida de la flor, cuando el estigma y las anteras hacen contacto al cerrarse ésta. Por otra parte, en días en que las flores reciben polinizadores que han visitado otras plantas previamente, habrá fertilización cruzada. El movimiento de estambres y estigmas resultando en la autogamia tardía ha sido documentado para otras especies. Por ejemplo, en Podophyllum hexandrum Royle (Berberidaceae), que responde de manera semejante a $T$. plumosum, donde la autogamia tardía es facilitada por el movimiento de los estambres hacia el estigma en el momento en que los pétalos empiezan a cerrarse (Xiong 
et al. 2013). En Aquilegia canadensis L. (Ranunculaceae) la autopolinización tardía es producto de la elongación progresiva de los estigmas hacia las anteras (Eckert \& Schaefer 1998). Shivanna (2012), recientemente también documentó el movimiento de los estigmas hacia las anteras en Passiflora edulis Sims (Passifloraceae), lo que promueve autogamia autónoma.

Es ampliamente conocido que la frecuencia de autoincompatibilidad en angiospermas se incrementa a medida que aumenta la longevidad de las especies. La frecuencia de auto-incompatibilidad es mayor en árboles $\mathrm{y}$ arbustos que en hierbas, y mayor en perennes que en anuales (Lloyd 1965, Arroyo \& Squeo 1990, Arroyo \& Uslar 1993, Raduski et al. 2012). Esto se corrobora con los datos actuales de sistemas de reproducción en la subfamilia Lomandroideae, que reflejan tendencias intrínsecas relacionadas con los rasgos de historia de vida. A la fecha se han evaluado algunas especies de los géneros Cordyline (leñosas) y Laxmannia (hierbas perennes). A diferencia de Trichopetalum, en Cordyline se ha reportado incompatibilidad genética en cuatro especies: Cordyline kaspar W.R.B. Oliv. (Beever 1981), C. pumilio Hook.f. (Beever 1983), C. australis (Forst.f.) Endl. (Beever \& Parkes 1996), y C. fruticosa (L.) A.Chev. (Hinkle 2007). Mientras que dos especies de Laxmannia han sido reportadas como autocompatibles (James et al. 1999).

El sistema reproductivo de T. plumosum es muy parecido al de Laxmannia ramosa Lindl. de Australia, donde hay autocompatibilidad y capacidad para autogamia autónoma en el momento en que las anteras hacen contacto con el estigma al cerrar la flor (James et al. 1999). Sin embargo, James et al. (1999) estudiaron una segunda especie de Laxmannia (L. sessiliflora Decne.) donde encontraron que si bien es autocompatible, no presenta autogamia tardía. Lloyd \& Schoen (1992) llevaron a cabo una revisión bibliográfica, donde concluyeron que las cantidades de autofecundación autónoma varían ampliamente entre especies y son influenciadas tanto por el grado de autocompatibilidad, la fenología y por las características de la morfología floral. Especies con un índice de autocompatibilidad alto, como es el caso de representantes de las familias: Balsaminaceae, Fabaceae, Lobeliaceae, Orchidaceae y Scrophulariaceae, carecen de autofertilidad total o tiene bajos índices de autofertilidad. Estas familias tienen mecanismos de polinización especializados y las flores presentan simetría bilateral, pétalos fusionados, y en la mayoría de las especies se encuentran pocos estambres por flor. Las especies con más del $50 \%$ de autofertilidad usualmente tienen flores con simetría radial, pétalos separados, y más de cinco estambres por flor (Lloyd \& Schoen 1992). Trichopetalum plumosum, cuyas características morfológicas florales concuerdan con esta última descripción, presenta un índice de autofertilidad alto $(\mathrm{IAF}=1,10)$ al compararlo con los datos reportados por Lloyd \& Schoen (1992), los resultados obtenidos confirman que la autofecundación autónoma es influenciada por las características de la morfología floral.

A futuro, sería interesante realizar comparaciones entre poblaciones que se encuentren en distintos hábitats a lo largo de la distribución de T. plumosum, para contrastar diferencias en el sistema reproductivo y documentar el posible cambio en la cantidad de autogamia autónoma relacionada con distintas condiciones bióticas (p.ej., variación en los ensambles de polinizadores) y/o abióticas (p.ej., precipitación y evapotranspiración potencial), generadas por el gradiente de aridez a lo largo de Chile central (Arroyo et al. 1995). Es muy probable que este gradiente de aridez latitudinal esté condicionando el sistema reproductivo, y se esperaría que en las poblaciones ubicadas al norte de la distribución de la especie donde el clima es menos húmedo, éstas presenten mayor autofecundación que en las poblaciones encontradas en el sur de su distribución.

\section{AGRADECIMIENTOS}

Agradecemos a los proyectos ICM-MINECON P05-002, PFB-23 por materiales y apoyo de beca doctoral para el primer año a MCD y de Magíster a ARC. A Beca CONICYTPCHA / Doctorado Nacional / 2015-21150912 otorgada a MCD. Al Departamento de Astronomía, Universidad de Chile, por el permiso para trabajar en Cerro Calán, y a los herbarios SGO, CONC y HULS. PJA agradece a Fondecyt Iniciación 11130299. A Cristian Ray, por la colaboración en terreno y laboratorio. También agradecemos a los revisores anónimos que ayudaron a mejorar de manera sustancial el documento.

\section{REFERENCIAS}

Aceituno, P. 1990. Anomalías climáticas en la región sudamericana durante los extremos de la Oscilación Austral. Revista Geofísica 32: 65-78.

APG III. 2009. An update of the Angiosperm Phylogeny Group classification for the orders and families of flowering plants: APG III. Botanical Journal of the Linnean Society 161(2): 105-121.

Arroyo, J., Barrett, S.C.H., Hidalgo, R., Cole, W.W. 2002. Evolutionary maintenance of stigma-height dimorphism in Narcissus papyraceus (Amaryllidaceae). American Journal of Botany 89(8): 1242-1249.

Arroyo, M.T.K., Squeo, F. 1990. Genetic self-incompatibility in the endemic Patagonian genus Benthamiella (Solanaceae). Gayana Botánica 47(1-2): 51-55.

Arroyo, M.T.K., Uslar, P. 1993. Breeding systems in a temperate mediterranean-type climate montane sclerophyllous forest in central Chile. Botanical Journal of the Linnean Society 111(1): 83-102.

Arroyo, M.T.K., Cavieres, L., Marticorena, C., Muñoz-Schick, M. 1995. Convergence in the mediterranean floras in 
Central Chile and California: insights from comparative biogeography. In: M.T.K. Arroyo, P.H. Zedler \& M.D. Fox (eds.), Ecology and biogeography of Mediterranean ecosystems in Chile, California, and Australia, pp. 43-88. Springer-Verlag, New York.

Barrett, S.C.H. 1998. The evolution of mating strategies in flowering plants. Trends in Plant Science 3(9): 335-341.

BARRETT, S.C.H. 2003. Mating strategies in flowering plants: the outcrossing-selfing paradigm and beyond. Philosophical Transactions of the Royal Society of London B 358: 9911004.

BarRetT, S.C.H., Harder, L.D. 1996. Ecology and evolution of plant mating. Trends in Ecology \& Evolution 11(2): 73-79.

Beever, R.E. 1981. Self-incompatibility in Cordyline kaspar (Agavaceae). New Zealand Journal of Botany 19(1): 1316.

Beever, R.E. 1983. Self-incompatibility in Cordyline pumilio (Agavaceae). New Zealand Journal of Botany 21(1): 9395.

BeEver, R.E., PARKes, S.L. 1996. Self-incompatibility in Cordyline australis (Asteliaceae). New Zealand Journal of Botany 34(1): 135-137.

Busch, J.W., Delph, L.F. 2012. The relative importance of reproductive assurance and automatic selection as hypotheses for the evolution of self-fertilization. Annals of Botany 109: 553-562.

Cavieres, L.A., Arroyo, M.T.K. 1999. Detección experimental de compatibilidad genética en la geófita Pasithea coerulea (Ruiz et Pavon) D. Don (Liliaceae). Gayana Botánica 56(1): 17-21.

Chase, M.W., Reveal, J.L., Fay, M.F. 2009. A subfamilial classification for the expanded asparagalean families Amaryllidaceae, Asparagaceae and Xanthorrhoeaceae. Botanical Journal of the Linnean Society 161(2): 132-136.

Di Castri, F., Hajek, E.R. 1976. Bioclimatología de Chile. Vicerrectoría Académica, Universidad Católica de Chile, Santiago. $128 \mathrm{pp}$.

Dirección Meteorológica de Chile. Dirección General de Aeronáutica Civil, Dirección Meteorológica de Chile, Sección Climatología. Anuario climatológico 2014. URL: http://www.meteochile.gob.cl/inicio.php Consultado: 10 de julio, 2015.

Eckert, C.G., Schaefer, A. 1998. Does self-pollination provide reproductive assurance in Aquilegia canadensis (Ranunculaceae)? American Journal of Botany 85(7): 919924.

EcKert, C.G., SAMIS, K.E., DART, S. 2006. Reproductive assurance and the evolution of uniparental reproduction in flowering plants. In: L.D. Harder \& S.C.H. Barrett (eds.), Ecology and evolution of flowers, pp. 183-203. Oxford University Press Inc., New York.

Eckert, C.G., Kalisz, S., Geber, M.A., Sargent, R., Elle, E., Cheptou, P.-O., Goodwillie, C., Johnston, M.O., Kelly, J.K., Moeller, D.A., Porcher, E., Ree, R.H., VallejoMarín, M., Winn, A.A. 2010. Plant mating systems in a changing world. Trends in Ecology and Evolution 25(1): 35-43.

Ferrer, M.M., Good, S.V. 2012. Self-sterility in flowering plants: preventing self-fertilization increases family diversification rates. Annals of Botany 110(3): 535-553.
Geerts, S., Pauw, A. 2012. The cost of being specialized: Pollinator limitation in the endangered geophyte Brunsvigia litoralis (Amaryllidaceae) in the Cape Floristic Region of South Africa. South African Journal of Botany 78: 159-164.

González, A.V., Pérez, F. 2010. Pollen limitation and reproductive assurance in the flora of the coastal Atacama Desert. International Journal of Plant Sciences 171(6): 607-614.

Goodwillie, C., Kalisz, S., EcKert, C.G. 2005. The evolutionary enigma of mixed mating systems in plants: occurrence, theoretical explanations, and empirical evidence. Annual Review of Ecology, Evolution, and Systematics 36: 47-79.

Guaglianone, E.R., Belgrano, M.J. 2003. Una nueva especie de Trichopetalum (Laxmanniaceae) y nueva cita del género para la Argentina. Hickenia 3(46): 189-194.

Hinkle, A.E. 2007. Population structure of Pacific Cordyline fruticosa (Laxmanniaceae) with implications for human settlement of Polynesia. American Journal of Botany 94(5): 828-839.

Hoffmann, A. 1989. Flora silvestre de Chile, zona central. Una guía para la identificación de las especies vegetales más frecuentes. Segunda Edición. Ediciones Fundación Claudio Gay, Santiago. 255 pp.

Holsinger, K.E. 2000. Reproductive systems and evolution in vascular plants. Proceedings of the National Academy of Sciences (USA) 97(13): 7037-7042.

IGIC, B., Busch, J.W. 2013. Is self-fertilization an evolutionary dead end? New Phytologist 198: 386-397.

Igic, B., Lande, R., Kohn, J.R. 2008. Loss of self-incompatibility and its evolutionary consequences. International Journal of Plant Sciences 169(1): 93-104.

James, S.H., Keighery, G.K., Moorrees, A., Waycott, M. 1999. Genomic coalescence in a population of Laxmannia sessiliflora (Angiospermae, Anthericaceae): an association of lethal polymorphism, self-pollination and chromosome number reduction. Heredity 82(4): 364-372.

Jara-Arancio, P., Arancio, G., Moreno, R., Carmona, M.R. 2006. Factores abióticos que influencian la germinación de seis especies herbáceas de la zona árida de Chile. Revista Chilena de Historia Natural 79: 309-319.

Jesson, L.K., BARRETT, S.C.H. 2002. Enantiostyly in Wachendorfia (Haemodoraceae): the influence of reproductive systems on the maintenance of the polymorphism. American Journal of Botany 89(2): 253-262.

Johnston, M.O., Porcher, E., Cheptou, P.-O., Eckert, C.G., Elle, E., Geber, M.A., Kalisz, S., Kelly, J.K., Moeller, D.A., VAllejo-Marín, M., Winn, A.A. 2009. Correlations among fertility components can maintain mixed mating in plants. The American Naturalist 173(1): 1-11.

Kalisz, S., Vogler, D.W. 2003. Benefits of autonomous selfing under unpredictable pollinator environments. Ecology 84(11): 2928-2942.

Kearns, C.A., Inouye, D.W. 1993. Techniques for pollination biologists. University Press of Colorado, USA. 583 pp.

Kleizen, C., Midgley, J., Johnson, S.D. 2008. Pollination systems of Colchicum (Colchicaceae) in southern Africa: evidence for rodent pollination. Annals of Botany 102(5): 747-755.

LARSON, B.M.H., BARRETT, S.C.H. 2000. A comparative analysis of pollen limitation in flowering plants. Biological Journal of the Linnean Society 69(4): 503-520.

Lloyd, D.G. 1965. Evolution of self-compatibility and 
racial differentiation in Leavenworthia (Cruciferae). Contributions from the Gray Herbarium of Harvard University 195: 3-134.

Lloyd, D.G., Schoen, D.J. 1992. Self- and cross-fertilization in plants. I. Functional dimensions. International Journal of Plant Sciences 153(3): 358-369.

Marais, W., ReIlly, J. 1978. Chlorophytum and its related genera (Liliaceae). Kew Bulletin 32(3): 653-663.

Muñoz, M., Moreira, A. 2000. Géneros endémicos de monocotiledóneas de Chile continental. URL: http://www. chlorischile.cl/Monocotiledoneas/trichopetalum gen.htm Consultado: 15 de julio, 2015.

NAvAs, L.E. 1973. Flora de la cuenca de Santiago de Chile. Tomo I. Pteridophyta, Gimnospermae, Monocotyledonae. Ediciones de la Universidad de Chile, Santiago, Chile. 301 pp.

Pérez, F., Arroyo, M.T.K., Armesto, J.J. 2009. Evolution of autonomous selfing accompanies increased specialization in the pollination system of Schizanthus (Solanaceae). American Journal of Botany 96(6): 1168-1176.

Raduski, A.R., Haney, E.B., Igić, B. 2012. The expression of self-incompatibility in angiosperms is bimodal. Evolution 66(4): 1275-1283.

Riedemann, P., Aldunate, G. 2001. Flora nativa de valor ornamental, identificación y propagación. Chile, zona centro. Editorial Andrés Bello. Santiago. 567 pp.

SaAvedra, F., Arroyo, M.T.K., Hoffmann, A.J. 1996. The breeding system of Hippeastrum advenum (Ker-Gawl.) Herb. (Amaryllidaceae), an endemic bulbous geophyte of the mediterranean-type climate region in central Chile.
Botanische Jahrbücher fur Systematik, Pflanzengeschichte und Pflanzengeographie 118(1): 1-8.

ShivanNA, K.R. 2012. Reproductive assurance through unusual autogamy in the absence of pollinators in Passiflora edulis (passion fruit). Current Science 103(9): 1091-1096.

Siegel, S., Castellan N.J. 1988. Nonparametric statistics for the behavioral sciences. Second Edition. McGraw-Hill, New York, USA. 399 pp.

StatSoft, Inc. 2004. STATISTICA (data analysis software system), version 7. www.statsoft.com.

Stevens, P.F. 2001 onwards. Angiosperm Phylogeny Website. Version 12, July 2012. URL: http://www.mobot.org/ MOBOT/research/APweb/Viewed: July 2, 2015.

Teillier, S., Aldunate, G., Riedemann, P., Niemeyer, H. 2005. Flora de la Reserva Nacional Río Clarillo: guía de identificación de especies. Impresos Socías Ltda. Chile. 367 pp.

Vogler, D.W., Kalisz, S. 2001. Sex among the flowers: the distribution of plant mating systems. Evolution 55(1): 202-204

Xiong, Y.-Z., Fang, Q., Huang, S.-Q. 2013. Pollinator scarcity drives the shift to delayed selfing in Himalayan mayapple Podophyllum hexandrum (Berberidaceae). AoB PLANTS 5: plt037; doi:10.1093/aobpla/plt037

ZAR, J.H. 2010. Biostatistical analysis. Fifth Edition. Prentice Hall, Inc., New Jersey, USA. 944 pp.

Zuloaga, F.O., Morrone, O., Belgrano, M.J. (eds.). 2008. Catálogo de las plantas vasculares del Cono Sur (Argentina, Sur de Brasil, Chile, Paraguay y Uruguay). Missouri Botanical Garden Press, St. Louis, Mo., USA. 3348 pp.

Recibido: 28.04 .2016

Aceptado: 03.10.2016 